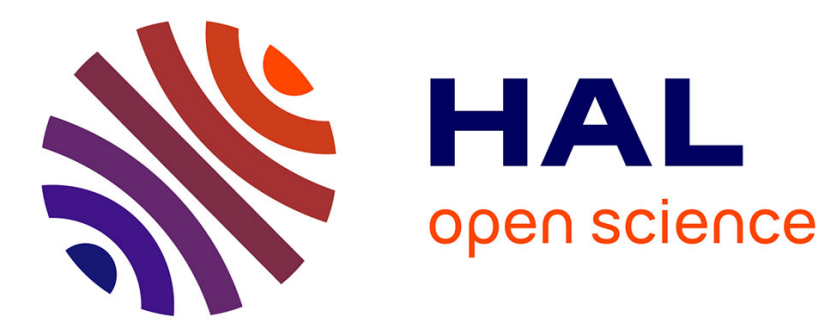

\title{
Slip length measurement of gas flow
}

Abdelhamid Maali, Stéphane Colin, Bharat Bhushan

\section{To cite this version:}

Abdelhamid Maali, Stéphane Colin, Bharat Bhushan. Slip length measurement of gas flow. Nanotechnology, 2016, 27 (37), pp.374004. 10.1088/0957-4484/27/37/374004 . hal-01392553

\section{HAL Id: hal-01392553 https://hal.science/hal-01392553}

Submitted on 4 Nov 2016

HAL is a multi-disciplinary open access archive for the deposit and dissemination of scientific research documents, whether they are published or not. The documents may come from teaching and research institutions in France or abroad, or from public or private research centers.
L'archive ouverte pluridisciplinaire HAL, est destinée au dépôt et à la diffusion de documents scientifiques de niveau recherche, publiés ou non, émanant des établissements d'enseignement et de recherche français ou étrangers, des laboratoires publics ou privés.

\section{다(1) (2)}

Distributed under a Creative Commons Attribution - ShareAlikel 4.0 International 


\title{
Slip length measurement of gas flow
}

\author{
Abdelhamid Maali ${ }^{1,2}$, Stéphane Colin $^{3}$ and Bharat Bhushan ${ }^{4}$ \\ ${ }^{1}$ Univ. Bordeaux, LOMA, UMR 5798, F 33400 Talence, France \\ ${ }^{2}$ CNRS, LOMA, UMR 5798, F 33400 Talence, France \\ ${ }^{3}$ Institut Clément Ader (ICA), Université de Toulouse, CNRS INSA ISAE Mines Albi UPS, Toulouse \\ France \\ ${ }^{4}$ Nanoprobe Laboratory for Bio \& Nanotechnology and Biomimetics, Ohio State University, 201 W 19th \\ Avenue, Columbus, OH 43210 1142, USA \\ E mail: bhushan.2@osu.edu
}

\begin{abstract}
In this paper, we present a review of the most important techniques used to measure the slip length of gas flow on isothermal surfaces. First, we present the famous Millikan experiment and then the rotating cylinder and spinning rotor gauge methods. Then, we describe the gas flow rate experiment, which is the most widely used technique to probe a confined gas and measure the slip. Finally, we present a promising technique using an atomic force microscope introduced recently to study the behavior of nanoscale confined gas.
\end{abstract}

Keywords: slip length, gas flow, confined gas, Millikan experiment, atomic force microscopy

\section{Introduction}

Understanding the physics of fluid flows at the micro- and nanoscale is crucial for designing, fabricating, and optimizing micro- and nano-electro-mechanical systems (MEMS/ NEMS). The large surface-to-volume ratio of MEMS/NEMS enables factors related to surface effects to dominate the fluid flow physics from the micro- to the nanoscale.

Fluid flow close to solid surfaces has been studied in detail in the last century [1 16]. In gases, due to local thermodynamic non-equilibrium, the velocity profile exhibits a phenomenon known as slip, which means that the fluid velocity near the solid surface is not equal to the velocity of the solid surface. The existence of slip in gas flows was first predicted by Maxwell [16] and its magnitude depends on the degree of rarefaction of the gas. To describe this degree of rarefaction, the Knudsen number, $K n=\lambda / D$, has been introduced. It compares the mean free path $\lambda$ of the gas molecules and a characteristic length $D$ of the flow domain, for example the hydraulic diameter of a duct. When the Knudsen number is on the order of 0.001 or larger, the rarefaction of the gas has to be taken into account, and a nonnegligible slip occurs on the surfaces [7 9, 12, 13]. The slip velocity has been initially modeled by Maxwell slip conditions for an isothermal wall as

$$
V_{s}=\left.b \frac{\partial V}{\partial z}\right|_{z=0} .
$$

In this equation, $b$ is the slip length. Maxwell proposed its form as $b=\lambda(2-\sigma) / \sigma$ where $\sigma$ is a coefficient that represents the fraction of the gas molecules that are reflected diffusively from the solid surface at $z=0$, assuming that the complementary fraction of the molecules is reflected specularly. The so-called slip velocity $V_{\mathrm{s}}$ is different from the actual macroscopic velocity of the gas at the wall: it is linearly extrapolated from the velocity profile outside the Knudsen layer (see figure 1), which is a very thin layer of gas in local thermal non-equilibrium state, where the usual Navier Stokes equations are not valid. The thickness of this layer is of the same order of magnitude as the mean free path $\lambda$. The initial expression (1) of the velocity slip provided by Maxwell, which is in a dimensionless form of the first order in $K n$, has further been discussed by numerous researchers, and various improved slip boundary conditions, including corrective coefficients and higher orders, have been proposed tentatively in the literature [17]. Unfortunately, there is currently no clear consensus for recommending the most appropriate equation and corrective coefficients for this slip boundary condition. In 


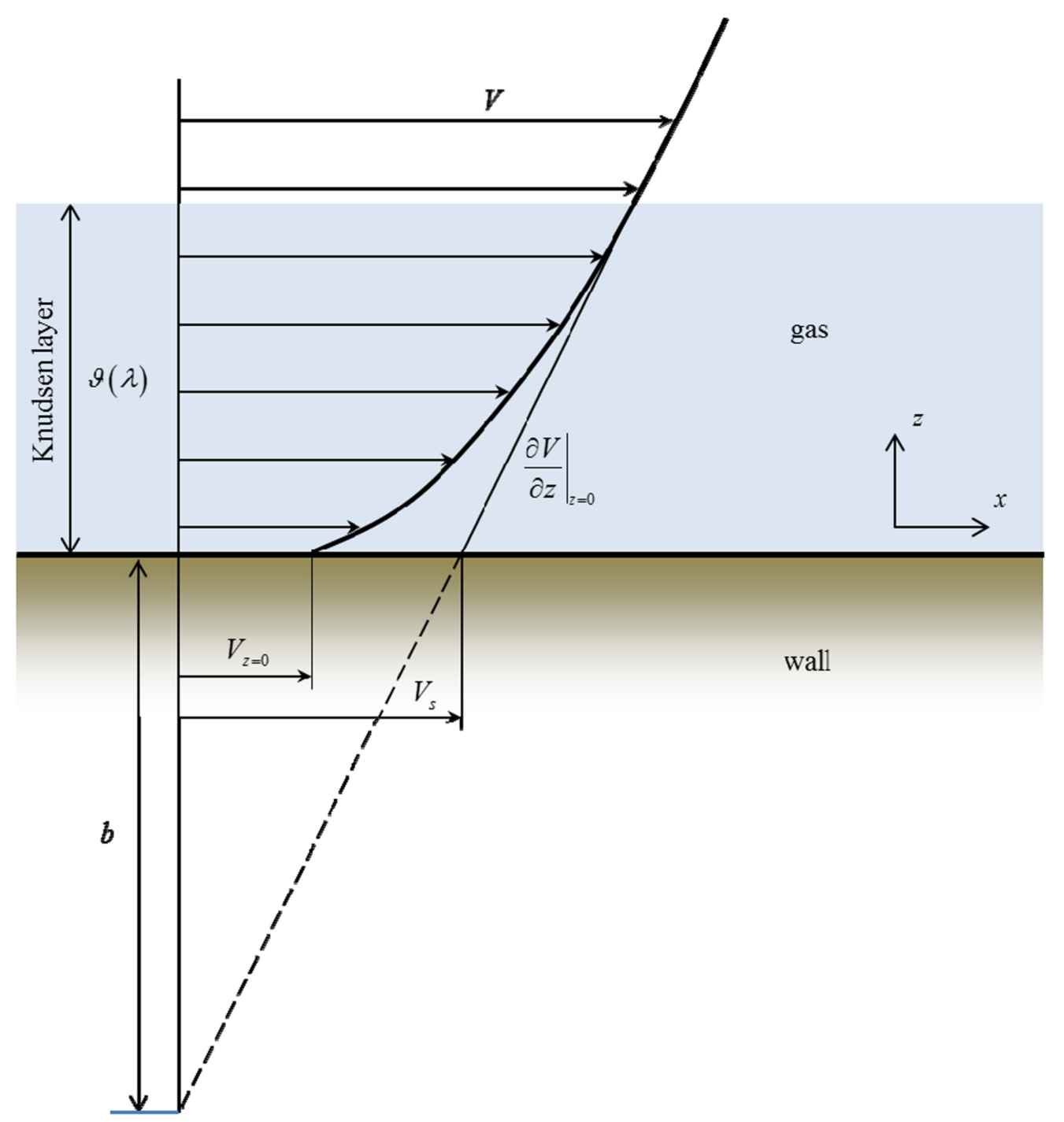

Figure 1. Knudsen layer and slip at the wall.

addition, there is often a confusion between $\sigma$ and the tangential momentum accommodation coefficient $\sigma_{t}$ [18]. The values of the coefficients $\sigma$ and $\sigma_{t}$ are also generally not well known.

Consequently, in order to discuss the accuracy and the limits of applicability of the boundary conditions proposed in the literature, there is a crucial need of smart experimental data on slip length in rarefied gas flows. In this paper, we review all techniques used to measure the slip length and the accommodation coefficient in a gas flow on isothermal surfaces. The paper is divided into five main sections. In section 2, the Millikan experiment is presented. In section 3, the rotating body techniques (rotating cylinder and spinning rotor gauge methods) are presented. Section 4 is devoted to mass flow rate measurements and velocimetry in micro- and nano-channels. In section 5, experimental results using an atomic force microscope (AFM) are shown. Finally, conclusions and the outlook for this research area are provided in section 6 .

\section{Millikan's droplet experiment}

Millikan has introduced the concept of gas slip to explain the results of his famous experiment on electron charge measurement [2, 19 21]. His experiment consists of measuring the terminal velocities $v_{1}$ and $v_{2}$ of a spherical charged droplet in two consecutive cases (see figure 2). In the first case, the droplet is falling with a speed $v_{1}$ under the influence of gravitational force, and in the second case, the droplet is rising against gravity with a speed $v_{2}$ in the presence of a vertically directed electric field $E$ [2, 19 21].

The terminal velocity in the absence of the electric field case is given by equating the Stokes force to the gravity force

$$
\frac{4}{3} \pi R^{3}(\mu-\rho) g=6 \pi \eta R v_{1}
$$

where $R$ is the droplet radius, $\eta$ is the air viscosity, $\mu$ is the droplet density, $\rho$ is the air density, and $g$ is the gravity acceleration. In the case where an electric field is applied, the 


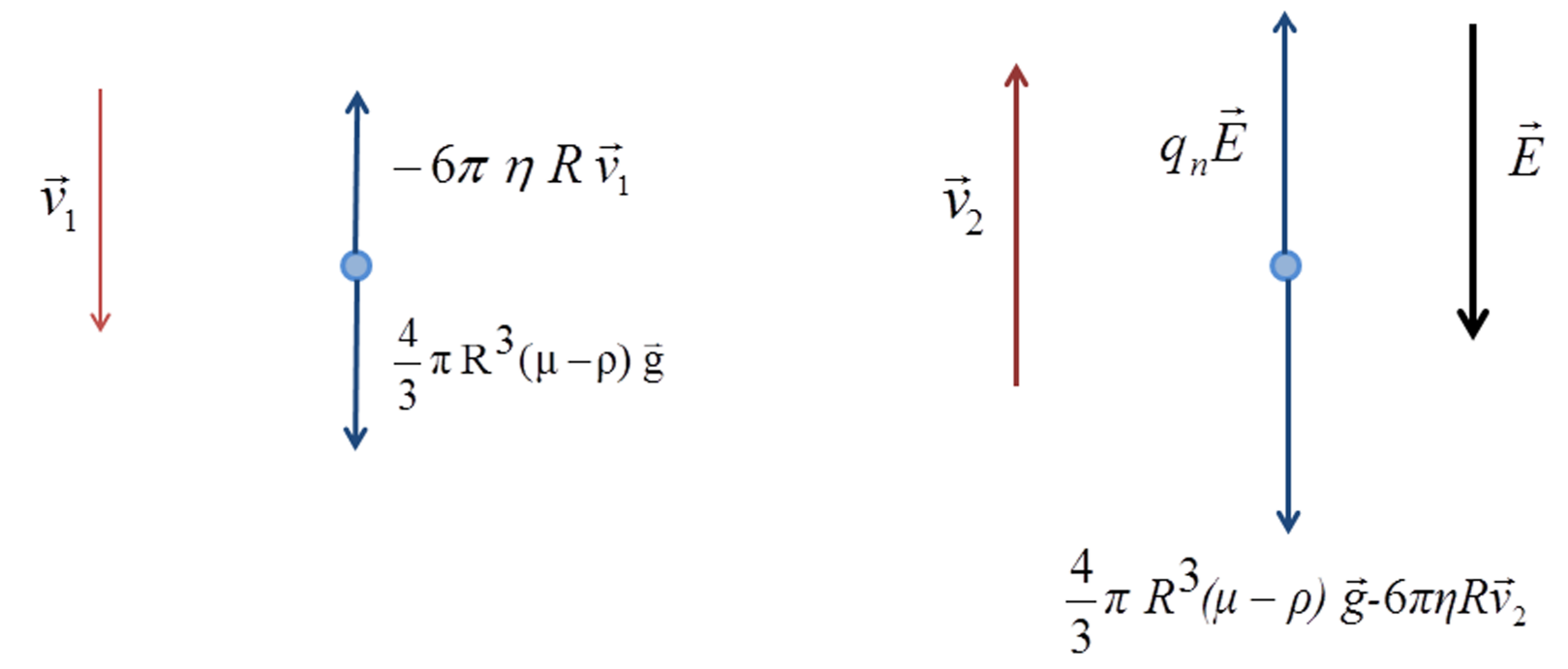

\section{A) Electric Field off}

B) Electric field on

Figure 2. (a) For a single drop carrying a negative charge $q_{n}$, if no electric field is present between the plates, the two forces acting on the charge are the force of gravity acting downward and a viscous drag force acting upward, as indicated in the figure. The drag force is proportional to the drop's speed. When the drop reaches its terminal speed $v_{1}$, the two forces balance each other. (b) If an electric field is applied between the plates such that the upper plate is at the higher electric potential, in this case, a force $q_{E}$ acts on the charged drop. Because $q$ is negative and $E$ is directed downward, this electric force is directed upward, as shown in the figure. If this force is sufficiently large, the drop moves upward and the drag force acts downward. When the upward electric force $q_{E}$ balances the sum of the gravitational force and the downward drag force, the drop reaches a new terminal speed $v_{2}$ in the upward direction.

terminal velocity is given by

$$
6 \pi \eta R v_{2}=-\frac{4}{3} \pi R^{3}(\mu-\rho) g+q_{n} E
$$

where $q_{n}$ is the electrical charge of the droplet. From the addition of equations (2) and (3) we obtain

$$
v_{1}+v_{2}=\frac{E q_{n}}{6 \pi \eta R}
$$

By eliminating the radius of the droplet from these equations, Millikan got the expression of the charge of the droplet as a function of the measured terminal velocities

$$
q_{n}=\frac{4 \pi}{3}\left(\frac{9 \eta}{2}\right)^{\frac{3}{2}} \frac{1}{\sqrt{g(\mu-\rho)}} \frac{v_{1}+v_{2}}{E} \sqrt{v_{1}}
$$

Millikan has shown that $\left(v_{1}+v_{2}\right)$ could be made to take on a series of values constituting an arithmetical progression, and having a greatest common divisor $\left(v_{1}+v_{2}\right)_{0}$, which corresponds to a charge of one electron upon the droplet, he got the equation for $e_{1}$, which is the value of the electric charge of the electron

$$
e_{1}=\frac{4 \pi}{3}\left(\frac{9 \eta}{2}\right)^{\frac{3}{2}} \frac{1}{\sqrt{g(\mu-\rho)}} \frac{\left(v_{1}+v_{2}\right)_{0}}{E} \sqrt{v_{1}} .
$$

The experimental results published by Millikan in 1911 show that $e_{1}$ did not come out at a constant value when droplets of different radii were used. A large droplet gives a consistently smaller value of $e_{1}$ than a smaller droplet.

Starting from his observation, he suggested taking into account a gas slip on the droplet surface during the motion. From the hydrodynamic concept, the slip at the droplet surface was taken by modifying the Stokes law $(6 \pi \eta R v)$, which becomes

$$
6 \pi \eta R v\left(\frac{1+2 b / R}{1+3 b / R}\right)
$$

which, for a small value of $b / R$, is reduced to

$$
\frac{6 \pi \eta R v}{1+b / R}
$$

where $b$ is, as described in his paper, the 'slip coefficient'. Millikan then obtained the relation between the extracted value of the electron charge $e_{1}$ from the measurements and the 


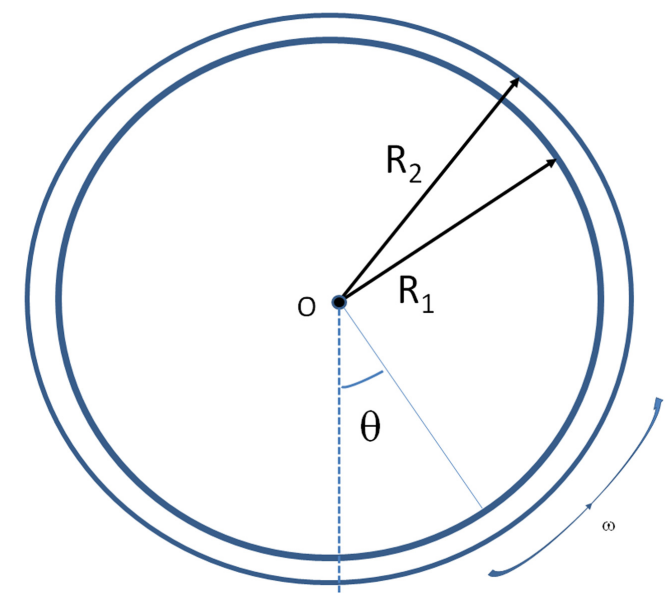

Figure 3. The inner cylinder has a radius $R_{1}$ and is suspended at position $O$. The deflection $\theta$ of the suspended cylinder is induced by the rotation of the cylinder $R_{2}$ at constant angular velocity $\omega$.

exact value of the electron charge $e$

$$
e\left(1+\frac{b}{R}\right)^{\frac{3}{2}}=e_{1}
$$

This equation shows how gas slips affect the measured value of the electron charge. The values extracted from larger droplets are less affected than those from smaller ones. The value of the slip length can be obtained by performing measurements of the electron charge versus the droplet radius.

The slip lengths measured by Millikan at temperature of $23{ }^{\circ} \mathrm{C}$ and at atmospheric pressure in air for droplets of oil, mercury, and shellac are respectively $79.3 \mathrm{~nm}, 66.7 \mathrm{~nm}$, and $101 \mathrm{~nm}$. These experimental results led Millikan to conduct a theoretical study of the slip length for different conditions of the reflection of molecules on surfaces. He showed that when molecules are reflected completely diffusively, a minimum of slip would result. The calculated minimum value of the slip in air at atmospheric pressure and at temperature of $23{ }^{\circ} \mathrm{C}$ is $66 \mathrm{~nm}$.

\section{Rotating body method}

\subsection{Rotating cylinder method}

The rotating cylinder method is one of the first methods used to measure the gas slip on surfaces. It has been employed by early researchers Millikan [2], Timiriazeff [22], Stacy [23], van Dyke [24], and Kuhlthau [25]. The method is based on using two concentric cylinders. The inner torsionally suspended cylinder has a radius $R_{1}$ (figure 3 ) and a length $L$, and the outer rotating cylinder a radius $R_{2}$.

The torsional moment is obtained from the measured angular deflection of the suspended cylinder $\theta$ by [2]

$$
\mathrm{M}=\frac{4 \pi^{2} I \theta}{T^{2}}
$$

where $I$ and $T$ are the moment of inertia and the oscillation period in vacuum of the suspended cylinder. If we assume no slip at either of the surfaces, the torsional moment that is acting on the suspended cylinder is given by

$$
\mathrm{M}=\frac{4 \pi^{2} I \theta}{T^{2}}=4 \pi L \eta \frac{R_{1}^{2} R_{2}^{2}}{R_{2}^{2}-R_{1}^{2}} \omega
$$

where $\omega$ is the angular velocity of the rotating outer cylinder and $\eta$ is the gas viscosity. When the slip becomes appreciable, the torsional moment expression as calculated by Millikan [2] is

$$
\begin{aligned}
\mathrm{M}= & \frac{4 \pi^{2} I \theta}{T^{2}}=4 \pi L \eta \frac{R_{1}^{2} R_{2}^{2}}{R_{2}^{2}-R_{1}^{2}} \\
& \times \frac{\omega}{1+2 b_{p}\left[\frac{R_{2}^{3}+R_{1}^{3}}{R_{1} R_{2}\left(R_{2}^{2}-R_{1}^{2}\right)}\right]}
\end{aligned}
$$

where $b_{p}$ is the slip length at pressure $p$.

At high pressure, the slip length is small. As a result, the effect of the slip on the torsional moment applied on the inner cylinder is negligible. In kinetic theory, viscosity is independent of pressure. So we can deduce that if the pressure is reduced in the experiment, the slip length will increase and the deflection of the inner cylinder should decrease. In practice, to determine the slip length by this method, it is necessary to measure the deflection $\theta$ at high pressure where the contribution of slip is negligible, and then reduce the pressure until the deflection has attained a measurable small value $\theta^{\prime}$. The slip coefficient for this low pressure value is then given by

$$
b_{p}=\left(\frac{\theta}{\theta^{\prime}}-1\right) \frac{R_{1} R_{2}\left(R_{2}^{2}-R_{1}^{2}\right)}{2\left(R_{2}^{3}+R_{1}^{3}\right)}
$$

Stacy [23] has used an apparatus with $R_{1}=5.43 \mathrm{~cm}$ and $R_{2}=6.06 \mathrm{~cm}$. For brass surfaces, he found that at atmospheric pressure and at room temperature, the slip length value is $b=66.1 \mathrm{~nm}$, which is practically the minimum value of the slip of the air calculated by Millikan [2] that corresponds to the case where all molecules are reflected diffusely after collision with the surfaces. For surfaces coated with shellac, Stacy has measured a slip length of $b=97 \mathrm{~nm}$ [23].

\subsection{Spinning rotor gauge method}

Another method was developed later [26 32] and called the spinning rotor gauge method, which has some similarity with the previous method in the sense that it is based on rotating techniques. The method is based on a body (disk or sphere) suspended magnetically and subjected to angular acceleration by means of magnetic force. Then, the disk or sphere is left to spin freely, and by measuring the subsequent angular deceleration velocity due to collision with the surrounding gas, the slip and accommodation coefficient can be obtained. 


\section{Gas flow rate measurement}

The measurement of slip length can be indirectly deduced from the flow rate measurement of rarefied gas flow through microchannels. This is a sound technique for flow in the slip flow regime, where the usual conservation equations (Navier Stokes equations) are valid and the flow rate is simply linked to the slip length. The technique can be accurate, provided that the dimensions of the microchannel, specifically of its cross-section, are known with high precision.

A similar approach can also be developed in large channels under low-pressure conditions, but the analogy is not complete between low pressure and micro gas flows. For the same value of the Knudsen number, a gas is more dilute at low pressure in a macrochannel than at higher pressure in a microchannel. In the former case, the validity of the ideal gas assumption that relies on a hypothesis of binary collisions between gas molecules is even better.

Whatever the operating conditions of the experiment (low pressure in a macrochannel or higher pressure in a microchannel), the flow rates to be measured in the slip flow regime remain much lower than what is accurately measurable with commercial gas flowmeters, typically limited to $10{ }^{8} \mathrm{~kg} \mathrm{~s}{ }^{1}$. Several specific experimental setups have been designed for measuring gas flow rates at low pressure, for an initial purpose of leak detection [33, 34] or to analyze pumping speed [35]. For example, the Physikalisch-Technische Bundesanstalt has designed a complex gas flowmeter that is able to measure gas flow rates between $4 \times 10^{-13} \mathrm{~mol} \mathrm{~s}^{-1}$ and $10^{-6} \mathrm{~mol} \mathrm{~s}^{-1}$ for very low pressure conditions, between $10^{-10} \mathrm{~Pa}$ and $3 \times 10^{-2} \mathrm{~Pa}$. Recently, improvements in microfabrication techniques have motivated researchers to develop specific setups dedicated to rarefied gas microflow measurements.

Two main kinds of solutions are now proposed in the literature:

- Several authors have developed a method based on the tracking of a liquid droplet [36 41]. In this method, the droplet is pushed by the gas flow in a calibrated pipette, and its tracking allows a direct measurement of the volume flow rate at the level of the droplet. However, this requires correction to obtain the mass flow rate through the microsystem, as shown below by equation (15).

- Other authors have developed indirect measurement techniques using the gas equation of state, generally assumed as an ideal gas. In temperature-regulated surroundings, mass flow rates can be deduced from the measurement of volume or pressure variations (with the so-called constant-pressure and constant-volume techniques, respectively). The constant-pressure technique is technologically difficult to implement, and it requires for example the use of a piston or a bellow controlled by an automation system to allow volume variation while maintaining a constant pressure [33, 35]. On the other hand, setups designed to implement the constant-volume technique are less complicated, as only pressure variation measurement inside a tank is required [41]. However, such systems could be very sensitive to thermal fluctuations.

- Some recent experimental setups are able to couple the two abovementioned methods [42], and some tests with the same microchannels have been conducted using different experimental setups in order to compare and discuss their accuracy [43].

For example, with the setup illustrated in figure 4 , the flow rate through a microsystem can be measured by the constant volume method or the droplet tracking method. This microsystem, which can be a simple microchannel or any more complex passive or active fluidic microsystem, is connected to valves V3A and V3B.

The constant-volume method can be implemented with valves V1A and V1B closed, while valves V2A, V2B, V3A, and $\mathrm{V} 3 \mathrm{~B}$ are open. The mass flow rate is then deduced from a double upstream and downstream pressure measurement as

$$
\dot{m}=-\frac{v_{A}}{R T} \frac{d p_{A}}{d t}=+\frac{v_{B}}{R T} \frac{d p_{B}}{d t}
$$

where $v_{A}$ and $v_{B}$ are the volume of reservoirs $\mathrm{A}$ and $\mathrm{B}$, respectively, including all tubing and fittings; $d p_{A} / d t$ and $d p_{B} / d t$ are the upstream and downstream pressure variations with time $t$, measured by highly sensitive capacitive pressure gauges CGA and CGB, respectively; $R$ is the specific gas constant; and $T$ is the uniform temperature controlled within the thermally-regulated setup.

If valves $\mathrm{V} 2 \mathrm{~A}$ and $\mathrm{V} 2 \mathrm{~B}$ are now closed, and a liquid droplet is placed in the calibrated pipettes in front of the optical sensors OSA and OSB, another way to get a double measure of the mass flow rate is from the equation

$$
\begin{aligned}
\dot{m} & =\frac{Q_{A}}{R T}\left\{p_{A i}-\delta p+p_{A i} \frac{v_{1 i}+Q_{A} \Delta t}{v_{A i}-Q_{A} \Delta t_{1}}\right\} \\
& =\frac{Q_{B}}{R T}\left\{p_{B i}+\delta p+p_{B i} \frac{v_{2 i}+Q_{B} \Delta t}{v_{B i}-Q_{B} \Delta t}\right\}
\end{aligned}
$$

where $Q_{A}$ and $Q_{B}$ are the volume flow rates deduced from the measurement of the upstream and downstream droplet displacement by the optical sensors OSA and OSB, respectively, during a short time $\Delta t$, and $\delta p$ is the pressure drop between both sides of the droplets. The initial volumes upstream from the droplet in pipette A, between this droplet and the microchannel, between the microchannel and the droplet in pipette $\mathrm{B}$, and downstream from this droplet are denoted by $v_{A i}, v_{1 i}, v_{2 i}$, and $v_{B i}$, respectively, and the initial pressures in reservoirs $\mathrm{A}$ and $\mathrm{B}$ are denoted by $p_{A i}$ and $p_{B i}$, respectively.

From the measurement of the mass flow rate, it is possible to extract the slip length, provided a specific slip boundary condition has been chosen. For example, considering a first-order slip boundary condition

$$
V_{s}=\left.b \frac{\partial V}{\partial r}\right|_{r=r_{0}}
$$

derived from Maxwell's boundary condition (1), the mass flow rate through a long microtube of radius $r_{0}$ and length $l$ 


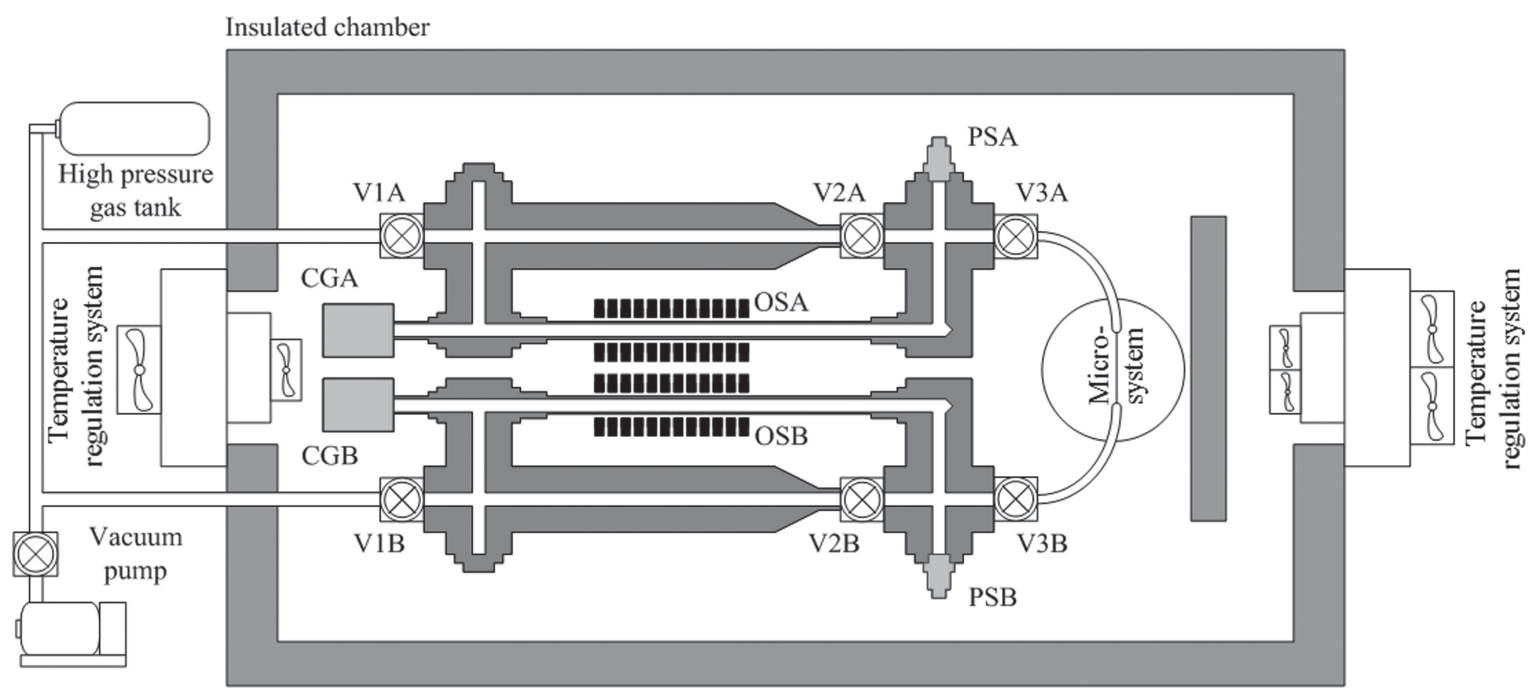

Figure 4. Example of experimental setup for mass flow rate measurement in microchannels using the droplet tracking and constant volume methods.

can be expressed as [44]

$$
\dot{m}=\frac{\pi r_{0}^{4} p_{B}^{2}}{4 \mu R T l}\left[\frac{\Pi^{2}-1}{4}+4 b K n_{B}(\Pi-1)\right]
$$

where $p_{B}$ is the outlet pressure, $\Pi=p_{A} / p_{B}$ is the ratio of the inlet over the outlet pressure, and $K n_{B}=\lambda_{B} /\left(2 r_{0}\right)$ is the outlet Knudsen number. Similar expressions can be derived for flows in rectangular microchannels and/or for more complex higher-order slip boundary conditions [45, 46]. A series of experimental measures of flow rates through microchannels (table 1) has been used by various authors $[9,39,40,42,43]$ with the objective to extract the value of the accommodation coefficient. Unfortunately, these values can hardly be directly compared because the theoretical model is often different from one author to another (based on either first-order or second-order boundary conditions, with or without correction from the Maxwell equation), the definition of the Knudsen number may vary, and the uncertainties are not always clearly estimated. In order to provide comparable results, all data in table 1 are provided as a function of the equivalent free path $\ell=\left(\mu_{g} / p\right) \sqrt{2 R T}$, as suggested by Sharipov [18]. This quantity does not represent the real mean free path, but provides an estimation directly calculated via measurable quantities, such as the pressure $p$, the temperature $T$, and the shear viscosity $\mu_{g}$ making its use easy in comparison to other ambiguous definitions. In table 1 , the Knudsen number $K n_{o}=\ell_{o} / D_{h}$ is then based on the equivalent free path $\ell_{o}$ at the outlet of the microchannel and on the hydraulic diameter $D_{h}$ of the microchannel crosssection. With the exception of the data from [47], which are the only data obtained using a commercial mass flowmeter, the value of the slip length found by specific setups has a narrow range between 1 and 1.4 times the equivalent free path, for various gases and material surfaces.

In order to get more detailed information on the slip length, some preliminary works have recently focused on velocimetry techniques able to provide not only the flow rate, but also the velocity profile within the microchannels. However, the first attempts using either micro particle image velocimetry ( $\mu \mathrm{PIV})$ [54], or micro molecular tagging velocimetry ( $\mu$ MTV) [55] have not been successfully extended yet to the slip flow regime, and direct measurement of slip at the wall with these techniques is still challenging, particularly due to the strong diffusion effects encountered at the microscale [56].

\section{AFM experiment}

The application of the AFM to the study of confined gas flow was motivated by recent experiments on liquid slip on solid surfaces using a contact AFM (Bonaccurso et al [57, 58]; Neto et al [59, 60]; Honing and Ducker [61]). The liquid boundary slip on non-wetting hydrophobic surfaces ensures that the drag force measured on such surfaces is always smaller than the one measured on hydrophilic wetting surfaces. The slip length value was extracted by fitting the experimental data using an expression calculated by Vinogradova [62]. In the AFM experiment the viscous force acting on the cantilever tip was measured during the approach at constant velocity to solid surfaces. These techniques were applied successfully to different surfaces and different liquids. However, the low viscosity of the gas (two orders of magnitude as low as the viscosity of water) made it difficult to measure the viscous drag force using a constant velocity of approach.

Maali and Bhushan [63] have used an AFM in dynamic mode to investigate the confined air flow close to solid surfaces at standard pressure. In their experiment, the air was confined between a glass surface and a spherical glass particle glued to an AFM cantilever. They used the amplitude modulation AFM mode where the amplitude and the phase of the cantilever vibration were measured versus the distance between the sphere and the surface, and then they extracted 
Table 1. Recent gas flow rate measurement through microchannels and extracted slip length $b$ as a function of the equivalent mean free path $\ell$.

\begin{tabular}{|c|c|c|c|c|}
\hline Reference & Channel material and section, experimental technique & Gas & $K n_{o}=\frac{\ell_{o}}{D_{h}}$ & $\frac{b}{\ell}$ \\
\hline \multirow[t]{3}{*}{ Arkilic et al 2001 [9] } & \multirow[t]{3}{*}{ Silicon, trapezoidal, constant volume method } & $\mathrm{N}_{2}$ & 0.0290 .197 & 1.25 \\
\hline & & $\mathrm{Ar}$ & 0.0290 .237 & 1.33 \\
\hline & & $\mathrm{CO}_{2}$ & 0.0170 .255 & 1.11 \\
\hline \multirow[t]{2}{*}{ Maurer et al 2003 [39] } & \multirow{2}{*}{ Pyrex silicon, rectangular, droplet tracking method } & $\mathrm{He}$ & 0.0950 .793 & 1.00 \\
\hline & & $\mathrm{N}_{2}$ & 0.0320 .669 & 1.13 \\
\hline \multirow[t]{2}{*}{ Colin et al 2004 [40] } & \multirow[t]{2}{*}{ Pyrex silicon, rectangular, droplet tracking method } & $\mathrm{He}$ & $0.018 \quad 0.131$ & 1.02 \\
\hline & & $\mathrm{N}_{2}$ & 0.0010 .054 & 1.02 \\
\hline Hsieh et al 2004 [47] & $\begin{array}{l}\text { Pyrex silicon, rectangular, commercial mass } \\
\text { flowmeter }\end{array}$ & $\mathrm{N}_{2}$ & 0.0070 .134 & 1.033 .14 \\
\hline \multirow[t]{3}{*}{ Ewart et al 2007 [48] } & \multirow[t]{3}{*}{ Fused silica, circular, constant volume method } & $\mathrm{He}$ & \multirow[t]{3}{*}{$<0.3$} & 1.001 .08 \\
\hline & & $\mathrm{Ar}$ & & 1.061 .21 \\
\hline & & $\mathrm{N}_{2}$ & & 0.981 .15 \\
\hline Ewart et al 2007 [49] & Pyrex silicon, trapezoidal, constant volume method & $\mathrm{He}$ & $<0.3$ & 1.151 .25 \\
\hline \multirow[t]{2}{*}{ Graur et al 2009 [50] } & \multirow[t]{2}{*}{ Pyrex silicon, trapezoidal, constant volume method } & $\mathrm{Ar}$ & \multirow{2}{*}{$<0.3$} & 1.141 .27 \\
\hline & & $\mathrm{N}_{2}$ & & 1.091 .11 \\
\hline \multirow{3}{*}{$\begin{array}{l}\text { Pitakarnnop et al } \\
2010 \text { [42] }\end{array}$} & \multirow[t]{3}{*}{ Pyrex silicon, rectangular, constant volume method } & $\mathrm{He}$ & 0.1250 .130 & 1.02 \\
\hline & & $\mathrm{Ar}$ & 0.0460 .047 & 1.02 \\
\hline & & $\mathrm{He}(30 \%) \operatorname{Ar}(70 \%)$ & order of 0.1 & 1.02 \\
\hline \multirow[t]{4}{*}{ Perrier et al 2011 [51] } & \multirow[t]{4}{*}{ Fused silica, circular, constant volume method } & $\mathrm{He}$ & $<0.3$ & 0.981 .05 \\
\hline & & $\mathrm{Xe}$ & & 1.091 .16 \\
\hline & & $\mathrm{Ar}$ & & 1.101 .14 \\
\hline & & $\mathrm{N}_{2}$ & & 1.101 .12 \\
\hline \multirow{3}{*}{$\begin{array}{l}\text { Yamaguchi et al } \\
2011 \text { [52] }\end{array}$} & \multirow[t]{3}{*}{ Fused silica, circular, constant volume method } & $\mathrm{Ar}$ & \multirow[t]{3}{*}{ order of 0.1} & 1.151 .39 \\
\hline & & $\mathrm{N}_{2}$ & & 1.121 .41 \\
\hline & & $\mathrm{O}_{2}$ & & 1.241 .41 \\
\hline $\begin{array}{l}\text { Yamaguchi et al } \\
2012[53]\end{array}$ & Stainless steel, circular, constant volume method & Ar, $\mathrm{N}_{2}, \mathrm{O}_{2}$ & order of 0.1 & 1.081 .15 \\
\hline Bergoglio et al 2015 [43] & $\begin{array}{l}\text { Glass silicon, rectangular, constant volume and } \\
\text { constant pressure methods }\end{array}$ & $\begin{array}{r}\mathrm{Ar}, \mathrm{N}_{2}, \mathrm{CO}_{2}, \mathrm{~N}_{2} \\
(95 \%) \mathrm{H}_{2}(5 \%)\end{array}$ & $<0.13$ & 1.02 \\
\hline
\end{tabular}

the damping and the stiffness of the interaction [63]. Due to the high quality factor of the cantilever far from the solid surface, the additional damping and stiffness induced by the hydrodynamic interaction during the confining of the gas were probed accurately.

For a cantilever oscillating with small amplitude, the interaction force acting on the cantilever can be linearized and has two contributions. The first is a conservative term $\left(-k_{H} z\right)$, resulting from the elastic compression of the film, and the second is a dissipative term $\left(-\gamma_{H} \dot{\mathrm{Z}}\right)$, resulting from viscous lubrication damping during the squeezing of the gas. The motion of the cantilever is then described by

$$
m^{*} \ddot{z}+\left(\gamma_{0}+\gamma_{H}\right) \dot{z}+\left(k_{l}+k_{H}\right) z=F_{0} \exp (j \omega t)
$$

where $m^{*}$ is the effective mass of the cantilever, $z$ is the instantaneous position of the cantilever, $k_{l}$ is the cantilever stiffness, and $\gamma_{0}$ is the bulk viscous damping far from the surface, and is related to the quality factor $Q$ and the resonance frequency $\omega_{0}$ via the equation

$$
Q=\frac{m^{*} \omega_{0}}{\gamma_{0}} \text {. }
$$

$k_{\mathrm{H}}$ and $\gamma_{\mathrm{H}}$ are the hydrodynamic interaction stiffness and damping coefficient, respectively, and $F_{0}$ is the driving force, which is given by

$$
F_{0}=\frac{k_{l} A_{0}}{Q}
$$

where $A_{0}$ is the amplitude of the oscillation far away from the interaction region (free amplitude of oscillation).

The steady-state solution $z=A \exp j(\omega t+\varphi)$ of equation (18) gives the stiffness and the damping coefficient:

$$
k_{H}=k_{l}\left[\frac{A_{0} \cos (\varphi)}{A Q}-1+\frac{\omega^{2}}{\omega_{0}^{2}}\right]
$$

and

$$
1+\gamma_{H} / \gamma_{0}=-\frac{\omega_{0} A_{0}}{\omega A} \sin (\varphi)
$$

where $A$ and $\varphi$ are the measured amplitude and phase of the oscillation, respectively, and $\omega$ is the driving frequency of the cantilever.

Figure 5(b) shows the measured amplitude and phase spectra of the cantilever far from the glass surface at a distance of $2000 \mu \mathrm{m}$ and at a distance of $3 \mu \mathrm{m}$. We can see from 

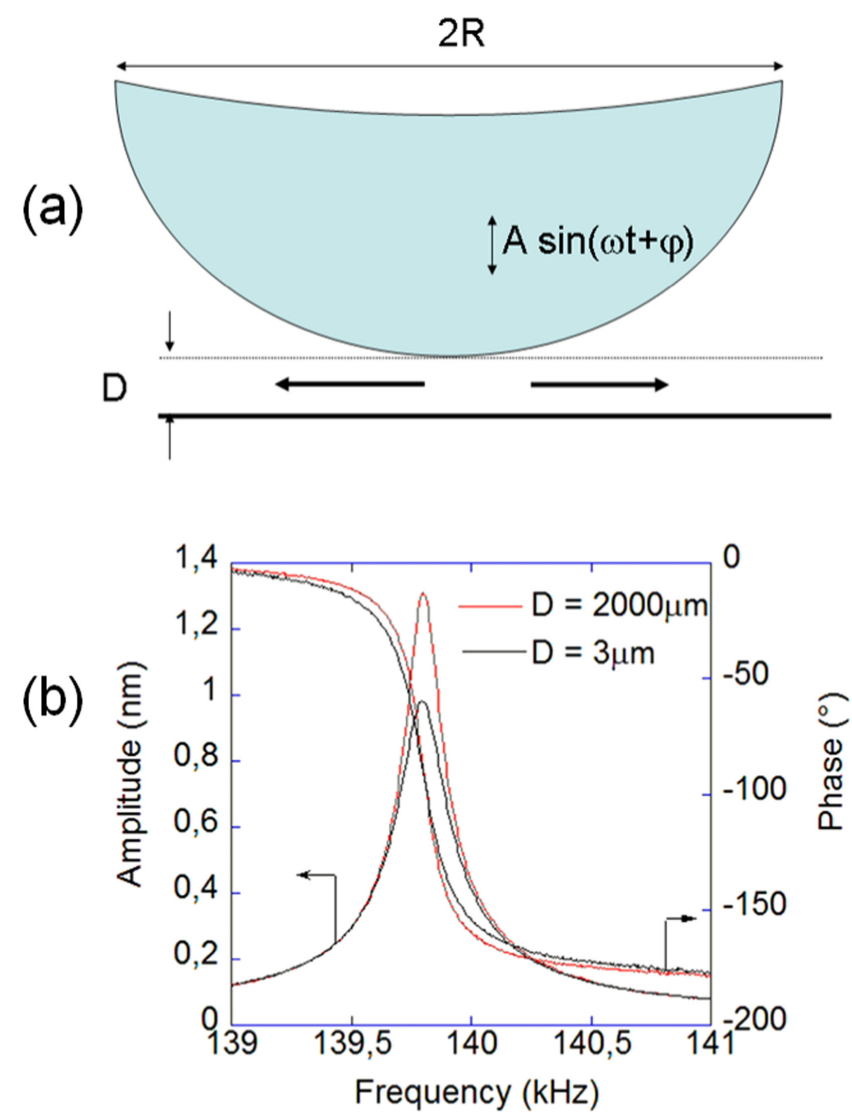

Figure 5. (a) Schematic illustration of a sphere vibrating close to a solid surface. (b) Amplitude and phase spectra of the cantilever obtained at two different positions from the glass surface (the dark lines are at $D=2000 \mu \mathrm{m}$ and the gray lines are at $D=3 \mu \mathrm{m}$ ).

this figure that when the gap between the sphere and the surface is reduced, the resonance frequency remains unchanged and only the amplitude and phase vary. To measure the induced viscous damping using the amplitude modulation mode, Maali and Bhushan [63] fixed the operating frequency and recorded the vibration amplitude and phase versus the distance.

Figure 6(a) shows the measured amplitude and phase of the cantilever as the gap between the glass sphere and the glass substrate is reduced. Figure 6(b) presents the DC deflection that allows one to determine the contact position of the two surfaces (zero distance) with accuracy better than $1 \mathrm{~nm}$. From the measured amplitude and phase signals they use equations (21) and (22) to extract the interaction stiffness and damping.

Figure 7 presents the damping coefficient $1+\gamma_{H} / \gamma_{0}$ and the stiffness $k_{H}$ versus the distance $D$. As the sphere approaches the glass surface, the damping coefficient increases due to the increase of the viscous drag force. However, the stiffness is unchanged and is equal to zero [63].

In the case of non-slip boundary conditions, the hydrodynamic force that acts on the sphere is given by the Taylor equation,

$$
F_{H}=\frac{6 \pi \eta R^{2}}{D} \dot{z}
$$
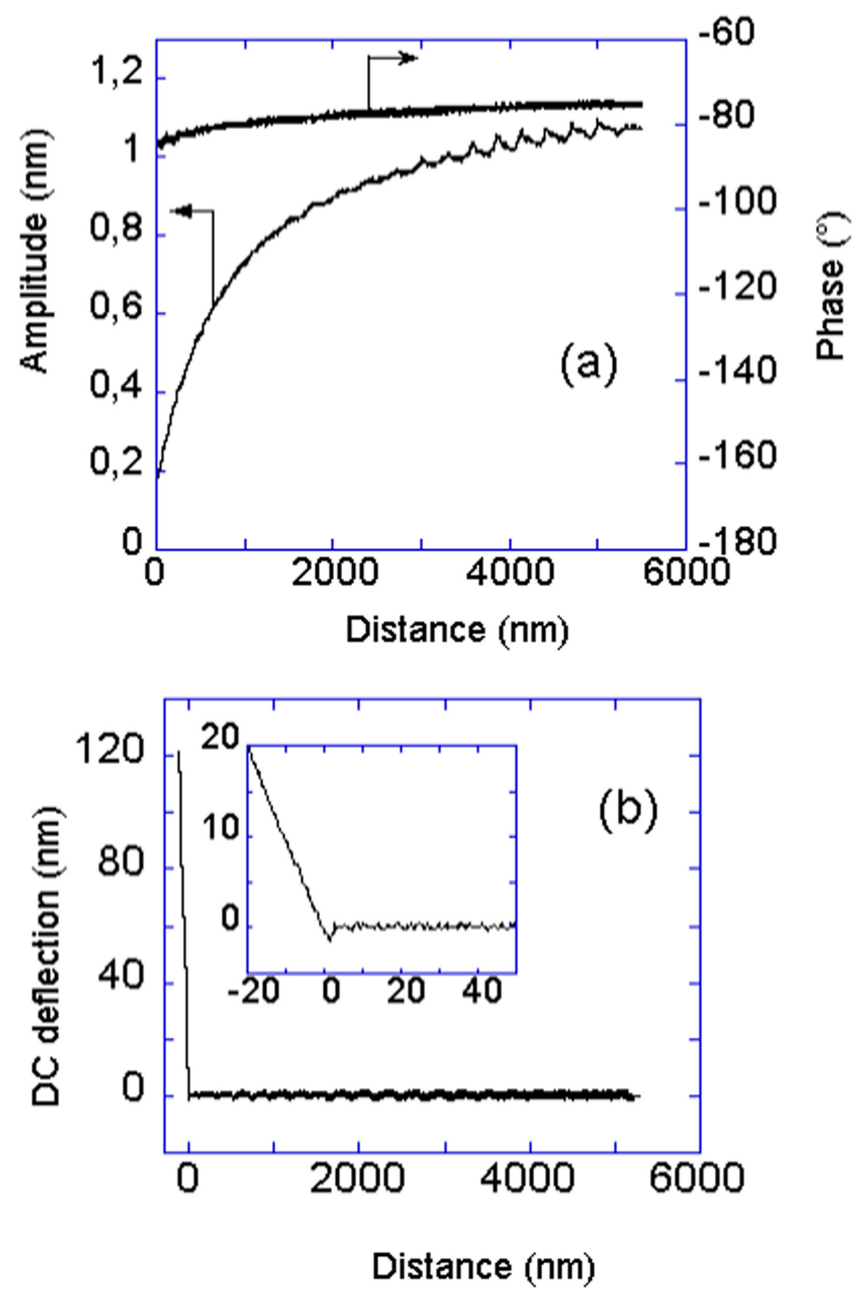

Figure 6. (a) The amplitude and phase of the cantilever measured as the glued sphere approaches the glass substrate. The oscillation frequency was fixed at $139.8 \mathrm{kHz}(20 \mathrm{~Hz}$ below the resonance frequency) and the oscillation amplitude far from the surface was $1.3 \mathrm{~nm}$. The modulation on the amplitude signal is due to interferences due to multiple reflections on both surfaces (the cantilever and substrate) of the laser light used to measure the cantilever motion. (b) The DC deflection of the cantilever measured as the glued sphere approaches the glass surface, which allows one to determine the contact position of the two surfaces. The inset shows a zoom of the DC deflection curve at a small distance [63].

where $R$ is the sphere radius, $D$ is the gap between the surfaces, $\eta$ is the viscosity of the air and $\dot{z}$ is the instantaneous velocity of the sphere. To take into account the boundary slip Vinogradova [62] introduced a correction function $f^{*}$ that depends on the distance $D$ and the slip length $b$ and showed that the expression of the viscous hydrodynamic force $F_{H}$ can be written as:

$$
F_{H}=\gamma_{H} \dot{z}=f^{*} \frac{6 \pi \eta R^{2}}{D} \dot{z}
$$

if both surfaces have the same slip length $b$

$$
f^{*}=\frac{2 D}{6 b}\left[\left(1+\frac{D}{6 b}\right) \ln \left(1+\frac{6 b}{D}\right)-1\right]
$$




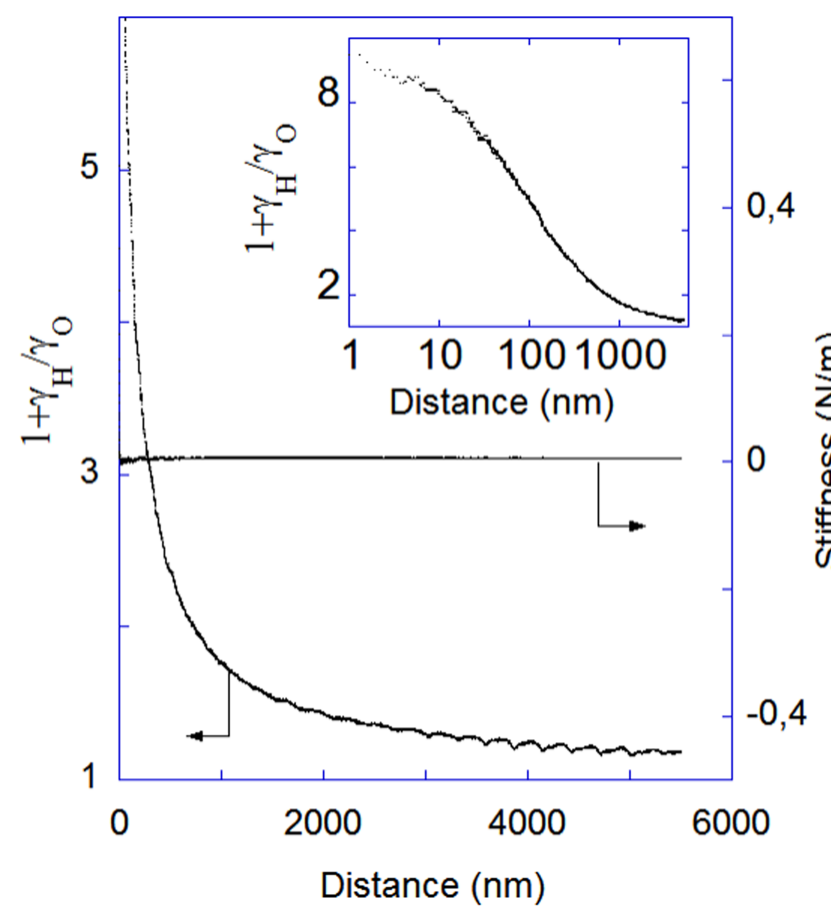

Figure 7. The normalized hydrodynamic damping $1+\gamma_{\mathbf{H}} / \gamma_{0}$ and the hydrodynamic interaction stiffness $\left(k_{\mathrm{H}}\right)$ versus the gap $(D)$ between the sphere and the glass substrate. The inset shows $1+\gamma_{\mathrm{H}} / \gamma_{0}$ versus the distance on a semilogarithmic plot [63].

The inverse of the normalized hydrodynamic damping is given as

$$
\gamma_{0} / \gamma_{H}=\frac{\gamma_{0}}{6 \pi \eta R^{2}} \frac{D}{f^{*}}
$$

At large separation distance $D \gg b, f^{*}$ can be expanded in series at the first order

$$
f^{*} \approx 1-\frac{2 b}{D}
$$

so, at large separation distance, the hydrodynamic force is

$$
\gamma_{0} / \gamma_{H} \approx \frac{\gamma_{0}}{6 \pi \eta R^{2}}(D+2 b)
$$

Figure 8 presents the inverse of the normalized hydrodynamic damping $\gamma_{0} / \gamma_{\mathrm{H}}$ obtained from the data presented in figure 7 . The linear extrapolation of the signal is represented by the dashed line. One can observe that the extrapolation curve does not pass through the origin, which indicates the presence of slip on the surfaces.

By fitting the data of the inverse of the normalized hydrodynamic damping using equation (26), Maali and Bhushan [63] obtained a slip length of $118 \pm 10 \mathrm{~nm}$. Furthermore, as shown in figure 8 the slip length $b$ can be extracted in this experiment without any need to fit parameters. The linear extrapolation of the inverse of the hydrodynamic damping versus the distance intersects the distance axis at a position of $2 b=236 \mathrm{~nm}$.

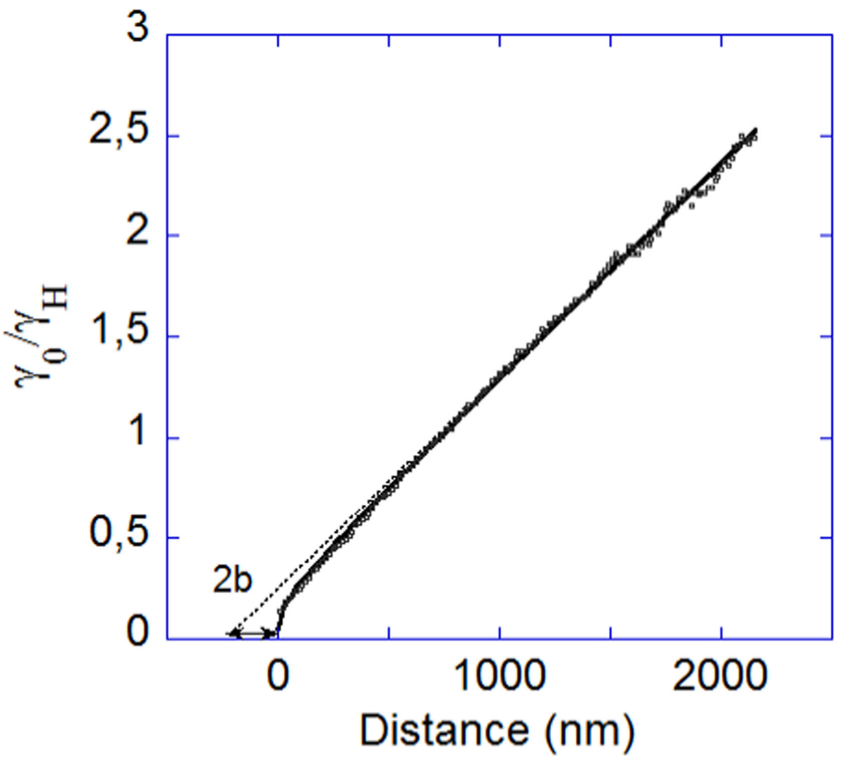

Figure 8. The inverse of the normalized hydrodynamic damping $\gamma_{0} /$ $\gamma_{\mathrm{H}}$ versus the distances between the sphere and the glass surface. The solid line is the fit curve using theoretical expression (equation (26)). The linear extrapolation of the signal is represented by the dashed line and it intersects the distance axis at position $2 \mathrm{~b}=236 \mathrm{~nm}[63]$.

For the air at atmospheric pressure and ambient temperature, the mean free path is $67 \mathrm{~nm}$ according to Bird [8], and thus, on the basis of the measured value of the slip length, the accommodation coefficient calculated using equation (1) is about $\sigma=0.72$.

Pan et al [64] have used the same techniques to measure the slip length on different surfaces at standard pressure. The measurements show that the slip length does not depend on the oscillation amplitude of the cantilever. Their data show that on glass, graphite, and mica surfaces the slip length is 98 $\pm 19 \mathrm{~nm}, 234 \pm 29 \mathrm{~nm}$, and $110 \pm 21 \mathrm{~nm}$, respectively. The adsorbed water on the surfaces causes inelastic collision allowing a greater accommodation coefficient (smaller slip length). The wettability of graphite surfaces is lower than that of mica and glass surfaces, and it may explain the high slip length and low accommodation coefficient.

Siria et al [65], Honig et al [66], Honig and Ducker [67], and Bowles and Ducker [68] have studied the thermally driven oscillation of a cantilever as it gradually approaches a wall. Under thermal excitation, the cantilever undergoes a vibration with amplitude that depends on the temperature and damping. In the approximation of a simple harmonic oscillator the power spectral density of the cantilever vibration is given by

$$
P S D(\omega)=\frac{2 k_{B} T \omega_{0}{ }^{3} / k_{l} Q(D)}{\left(\omega^{2}-\omega_{0}^{2}\right)^{2}+\omega^{2} \omega_{0}^{2} / Q(d)^{2}}
$$

where $k_{B}$ is the Boltzmann constant, $T$ is the temperature and $Q(d)$ is the cantilever quality factor at distance $d$ from the surface. The damping at distance $d$ is obtained from the 
quality factor by

$$
\gamma(d)=\frac{k_{l}}{\omega_{0} Q(d)}
$$

From an analysis of measurements of the damping versus the distance, Siria et al [65] reported perfect boundary slip conditions for the air flow between a silicon cantilever and a flat cleaved optical fiber. Honig et al [67], Honig and Ducker [66], and Bowles and Ducker [68] have used a similar technique (thermal excitation) and shown an opposite result. They measured a finite slip in agreement with the results of Maali and Bhushan [63], with a slip value ranging between 100 and $630 \mathrm{~nm}$ depending on the nature of the surfaces and their preparations.

Let us note that, in AFM experiments, the Knudsen number can be varied continuously over several decades by varying the distance between the two surfaces. As the distance is reduced (increasing the Knudsen number) the cantilever damping increases due to the viscous flow of confined gas between the surfaces. The measured damping is described by the continuous hydrodynamic approach that takes into account the slip of the gas on the surfaces.

\section{Conclusion and outlook}

We have described in this paper the most important techniques used to measure the slip length and the accommodation coefficient in a gas flow on isothermal surfaces. In all of the presented techniques, the gas slip is measured indirectly. The slip length related to the slip velocity is extracted from the measurement of the velocity of the drop (Millikan method), the deflection (rotating cylinder), the mass flow rate (mass flow rate experiment), and the force measurement (AFM experiment).

Particle image velocimetry, which was used successfully in the last decades to directly measure liquid slip close to solid surfaces [69, 70], was adopted to study gas flows. Some preliminary works have recently focused on this technique within gas microchannels. However, the first attempts using either $\mu \mathrm{PIV}$ [54] or $\mu \mathrm{MTV}$ [55] have not been successfully extended yet to the slip flow regime, and direct measurement of slip at the wall with these techniques is still challenging, particularly due to the strong diffusion effects encountered at the microscale [56].

Recent results of the Ducker group have shown that it is possible to modify the gas slip and the accommodation coefficients in situ [71]. By increasing the temperature of their surface from $18{ }^{\circ} \mathrm{C}$ to $40{ }^{\circ} \mathrm{C}$, they showed that the slip increases from $290 \mathrm{~nm}$ to $500 \mathrm{~nm}$. They explained the increase of the slip by the modification of the roughness of their surface, which opens the way to in situ control of gas flow on a given surface.

In this paper the surfaces are assumed isothermal; however there are some situations where the temperature is not uniform. In such cases, the temperature gradient gives rise to the so-called thermal creep that induces a lateral flow velocity of the gas. Understanding and optimization of this effect may open the way to the realization of gas micro-pumps controlled by temperature gradients.

\section{References}

[1] Maxwell J C 1879 On stresses in rarefied gases arising from inequalities of temperature Phil. Tran. R. Soc. 170 23156

[2] Millikan R A 1923 Coefficients of slip in gases and the law of reflection of molecules from the surfaces of solids and liquids Phys. Rev. 2121738

[3] Meyer O E 1866 Pogg. Ann. 127269

[4] Knudsen M 1909 Die Gesetze der Molekularströmung und der inneren Reibungsströmung der Gase durch Röhren Ann. d. Physik 2875

[5] Fisher W J 1909 The molecular and the frictional flow of gases in tubes Phys. Rev. 29325

[6] Morris D L, Hannon L and Garcia A L 1992 Slip length in a dilute gas Phys. Rev. A 46527981

[7] Veijola T, Kuisma H, Lahdenperä J and Ryhänen T 1995 Equivalent circuit model of the squeezed gas film in a silicon accelerometer Sens. Actuators, A Phys. 4823948

[8] Bird G A 1998 Molecular Gas Dynamics and the Direct Simulation of Gas Flows (Oxford: Clarendon Press)

[9] Arkilic E B, Breuer K S and Schmidt M A 2001 Mass flow and tangential momentum accommodation in silicon micromachined channels J. Fluid Mech. 4372943

[10] Lockerby D A, Reese J M, Emerson D R and Barber R W 2004 Velocity boundary condition at solid walls in rarefied gas calculations Phys. Rev. E 70017303

[11] Cao B Y, Chen M and Guo Z Y 2005 Temperature dependence of the tangential momentum accommodation coefficient for gases Appl. Phys. Lett. 86091905

[12] Bao M and Yang H 2007 Squeeze film air damping in MEMS Sens. Actuators A Phys. 136327

[13] Cooper S M, Cruden B A, Meyyappan M, Raju R and Roy S 2004 Gas transport characteristics through a carbon nanotubule Nano Lett. 437781

[14] Bhushan B 2007 Springer Handbook of Nanotechnology 2nd edn (Heidelberg, Germany: Springer)

[15] Lauga E, Brenner M P and Stone H A 2007 Microfluidics: the no slip boundary condition Handbook of Experimental Fluid Dynamics ed C Tropea, A Yarin and J F Foss (New York: Springer)

[16] Maxwell J C 1867 On the dynamical theory of gases Phil. Trans. R. Soc. 1574988

[17] Colin S 2005 Rarefaction and compressibility effects on steady and transient gas flows in microchannels Microfluid. Nanofluid. 126879

[18] Sharipov F 2011 Data on the velocity slip and temperature jump on a gas solid interface J. Phys. Chem. Ref. Data 40 023101

[19] Millikan R A 1911 The isolation of an ion, a precision measurement of its charge, and the correction of Stokes's law Phys. Rev. (Series I) 32349

[20] Millikan R A 1913 On the elementary electrical charge and the Avogadro constant Phys. Rev. 2109

[21] Millikan R A 1923 The general law of fall of a small spherical body through a gas, and its bearing upon the nature of molecular reflection from surfaces Phys. Rev. 221

[22] Timiriazeff A 1913 ber die innere Reibung verdnnter Gase und ber den Zusammenhang der Gleitung und des Temperatursprunges an der Grenze zwischen Metall und Gas Ann. Phys. 34597191 
[23] Stacy L J 1923 A determination by the constant deflection method of the value of the coefficient of slip for rough and for smooth surfaces in air Phys. Rev. 2123949

[24] van Dyke K S 1923 The coefficients of viscosity and of slip of air and of carbon dioxide by the rotating cylinder method Phys. Rev. 2125065

[25] Kuhlthau A R 1949 Air friction on rapidly moving surfaces J. Appl. Phys. 2021723

[26] Comsa G, Fremerey J K, Lindenau B, Messer G and Rohl P 1980 Calibration of a spinning rotor gas friction gauge against a fundamental vacuum pressure standard $J$. Vac. Sci. Technol. 176424

[27] Gabis D S, Loyalka S K and Strovick T S 1996 Measurements of the tangential momentum accommodation coefficient in the transition flow regime with a spinning rotor gauge J. Vac. Sci. Technol. A 1425928

[28] Tekasakul P, Bentz J A, Tompson R V and Loyalka S K 1996 The spinning rotor gauge: measurements of viscosity, velocity slip coefficients, and tangential momentum accommodation coefficients J. Vac. Sci. Technol. A 14294652

[29] Bentz J A, Tompson R V and Loyalka S K 1997 The spinning rotor gauge: measurements of viscosity, velocity slip coefficients, and tangential momentum accommodation coefficients for N2 and CH4 Vacuum 4881724

[30] Bentz J A, Tompson R V and Loyalka S K 2001 Measurements of viscosity, velocity slip coefficients, and tangential momentum accommodation coefficients using a modified spinning rotor gauge J. Vac. Sci. Technol. A 1931724

[31] Jousten K 2003 Is the effective accommodation coefficient of the spinning rotor gauge temperature dependent? J. Vac. Sci. Technol. A 2131824

[32] Gronych T, Ulman R, Peksa L and Repa P 2004 Measurements of the relative tangential momentum accommodation coefficient for different gases with a viscosity vacuum gauge Vacuum 732759

[33] McCulloh K E, Tilford C R, Ehrlich C D and Long F G 1987 Low range flowmeters for use with vacuum and leak standards J. Vac. Sci. Technol. A 537681

[34] Bergoglio M, Calcatelli A and Rumiano G 1995 Gas flowrate measurements for leak calibration Vacuum 467635

[35] Jousten K, Menzer H and Niepraschk R 2002 A new fully automated gas flowmeter at the PTB for flow rates between $1013 \mathrm{~mol} \mathrm{~s}^{-1}$ and $106 \mathrm{~mol} \mathrm{~s}^{-1}$ Metrologia 3951929

[36] Pong K C, Ho C M, Liu J and Tai Y C 1994 Non linear pressure distribution in uniform microchannels Application of Microfabrication to Fluid Mechanics ed P R Bandyopadhyay, K S Breuer and C J Blechinger (New York: ASME) pp 516

[37] Harley J C, Huang Y, Bau H H and Zemel J N 1995 Gas flow in micro channels J. Fluid Mech. 28425774

[38] Zohar Y, Lee S Y K, Lee W Y, Jiang L and Tong P 2002 Subsonic gas flow in a straight and uniform microchannel J. Fluid Mech. 47212551

[39] Maurer J, Tabeling P, Joseph P and Willaime H 2003 Second order slip laws in microchannels for helium and nitrogen Phys. Fluids 15261321

[40] Colin S, Lalonde P and Caen R 2004 Validation of a second order slip flow model in rectangular microchannels Heat Transfer Eng. 252330

[41] Ewart T, Perrier P, Graur I and Méolans J G 2006 Mass flow rate measurements in gas micro flows Exp. Fluids 4148798

[42] Pitakarnnop J, Varoutis S, Valougeorgis D, Geoffroy S, Baldas L and Colin S 2010 A novel experimental setup for gas microflows Microfluid. Nanofluid. 85772

[43] Bergoglio M, Mari D, Chen J, Si Hadj Mohand H, Colin S and Barrot C 2015 Experimental and computational study of gas flow delivered by a rectangular microchannels leak Measurement 7355162
[44] Kandlikar S G, Garimella S, Li D, Colin S and King M R 2013 Heat Transfer and Fluid Flow in Minichannels and Microchannels 2nd edn (Oxford: Elsevier)

[45] Kandlikar S G, Colin S, Peles Y, Garimella S, Pease R F, Brandner J J and Tuckerman D B 2013 Heat transfer in microchannels 2012 status and research needs J. Heat Transf. Trans. ASME 135091001

[46] Aubert C and Colin S 2001 High order boundary conditions for gaseous flows in rectangular microchannels Microscale Thermophys. Eng. 54154

[47] Hsieh S S, Tsai H H, Lin C Y, Huang C F and Chien C M 2004 Gas flow in a long microchannel Int. J. Heat Mass Transf. 47387787

[48] Ewart T, Perrier P, Graur I and Méolans J G 2007 Tangential momemtum accommodation in microtube Microfluid. Nanofluid. 368995

[49] Ewart T, Perrier P, Graur I A and Méolans J G 2007 Mass flow rate measurements in a microchannel, from hydrodynamic to near free molecular regimes J. Fluid Mech. 58433756

[50] Graur I, Perrier P, Ghozlani W and Meolans J 2009 Measurements of tangential momentum accommodation coefficient for various gases in plane microchannel Phys. Fluids 21102004

[51] Perrier P, Graur I A, Ewart T and Méolans J G 2011 Mass flow rate measurements in microtubes: from hydrodynamic to near free molecular regime Phys. Fluids 23042004

[52] Yamaguchi H, Hanawa T, Yamamoto O, Matsuda Y, Egami Y and Niimi T 2011 Experimental measurement on tangential momentum accommodation coefficient in a single microtube Microfluid. Nanofluid. 115764

[53] Yamaguchi H, Matsuda Y and Niimi T 2012 Tangential momentum accommodation coefficient measurements for various materials and gas species J. Phys.: Conf. Series 362 012035

[54] Yoon S Y, Ross J W, Mench M M and Sharp K V 2006 Gas phase particle image velocimetry (PIV) for application to the design of fuel cell reactant flow channels J. Power Sources 160101725

[55] Samouda F, Colin S, Barrot C, Baldas L and Brandner J J 2015 Micro molecular tagging velocimetry for analysis of gas flows in mini and micro systems Microsyst. Technol. 21 52737

[56] Frezzotti A, Si Hadj Mohand H, Barrot C and Colin S 2015 Role of diffusion on molecular tagging velocimetry technique for rarefied gas flow analysis Microfluid. Nanofluid. 19133548

[57] Bonaccurso E, Kappl M and Butt H J 2002 Hydrodynamic force measurements: boundary slip of water on hydrophilic surfaces and electrokinetic effects Phys. Rev. Lett. 88 076103

[58] Bonaccurso E, Butt H J and Craig V S J 2003 Surface roughness and hydrodynamic boundary slip of a newtonian fluid in a completely wetting system Phys. Rev. Lett. 90 144501

[59] Neto C, Craig V S J and Williams D R M 2003 Evidence of shear dependent boundary slip in newtonian liquids Eur. Phys. J. E 12 S71

[60] Neto C, Evans D R, Bonaccurso E, Butt H J and Craig V S J 2005 Boundary slip in newtonian liquids: a review of experimental studies Rep. Prog. Phys. 682859

[61] Honig C D F and Ducker W A 2007 No slip hydrodynamic boundary condition for hydrophilic particles Phys. Rev. Lett. 98028305

[62] Vinogradova O I 1995 Drainage of a thin liquid film confined between hydrophobic surfaces Langmuir 112213

[63] Maali A and Bhushan B 2008 Slip length measurement of confined air flow using dynamic atomic force microscopy Phys. Rev. E 78027302 
[64] Pan Y, Bhushan B and Maali A 2013 Slip length measurement of confined air flow on three smooth surfaces Langmuir 294298

[65] Siria A, Drezet A, Marchi F, Comin F, Huant S and Chevrier J 2009 Viscous cavity damping of a microlever in a simple fluid Phys. Rev. Lett. 102254503

[66] Honig C D F and Ducker W A 2010 Effect of molecularly thin films on lubrication forces and accommodation coefficients in air J. Phys. Chem. C 114201149

[67] Honig C D F, Sader J E, Mulvaney P and Ducker W A 2010 Lubrication forces in air and accommodation coefficient measured by a thermal damping method using an atomic force microscope Phys. Rev. E 81056305
[68] Bowles A and Ducker W 2011 Gas flow near a smooth plate Phys. Rev. E 83056328

[69] Ou J and Rothstein J P 2005 Direct velocity measurements of the flow past drag reducing ultrahydrophobic surfaces Phys. Fluids 17103606

[70] Joseph P, Cottin Bizonne C, Benoît J M, Ybert C, Journet C, Tabeling P and Bocquet L 2006 Slippage of water past superhydrophobic carbon nanotube forests in microchannels Phys. Rev. Lett. 97156104

[71] Seo. D and Ducker W A 2013 In situ control of gas flow by modification of gas solid interactions PRL 111 174502 